\title{
Estudo de composições cerâmicas à base de alumina e vitrocerâmico do sistema LZSA para obtenção de estruturas multicamadas por tape casting
}

\section{(Study of ceramic compositions based on alumina and LZSA glass-ceramic for obtaining multilayer structures by tape casting)}

\author{
F. D. Minatto, E. da S. Alexandre, A. De Noni Jr., O. R. K. Montedo \\ Laboratório de Cerâmica Técnica, Programa de Pós-graduação em Ciência e Engenharia de Materiais, \\ Universidade do Extremo Sul Catarinense, Av. Universitária, 1105, Criciúma, SC, Brasil 88806-000 \\ flavia.dagostimminatto@gmail.com,elizandra.alexandre@hotmail.com, \\ agenordenoni@gmail.com,oscar.rkm@gmail.com
}

\begin{abstract}
Resumo
Compósitos de matriz cerâmica têm sido desenvolvidos nas últimas décadas como um meio eficiente de melhoria da tenacidade à fratura. Compósitos cerâmicos multicamadas se destacam por seu relativo baixo custo e facilidade de obtenção. Estes compósitos são confeccionados com camadas alternadas de dois materiais cerâmicos e têm como mecanismo de tenacificação o desvio de trajetória de trincas em propagação, camada após camada, no compósito. Neste trabalho foram estudadas composições à base de alumina e vitrocerâmico $\mathrm{LZSA}_{(\mathrm{Li}} \mathrm{O}_{-} \mathrm{ZrO}{ }_{2}-$ $\mathrm{SiO}_{2}-\mathrm{Al}_{2} \mathrm{O}_{3}$ ) para compor as camadas a fim de se obter compósito multicamada com interfaces fortes. Assim, tensões residuais compressivas nas camadas finas à base de LZSA intercaladas às camadas à base de $\mathrm{Al}_{2} \mathrm{O}_{3}$, sob leve tensão residual de tração, foram esperadas obter. As composições foram definidas por planejamento experimental fatorial, gerando corpos de prova por prensagem, os quais foram sinterizados e caracterizados quanto ao coeficiente de expansão térmica e módulo de elasticidade. Três composições (\% em massa) à base de alumina (CA1: $100 \% \mathrm{Al}_{2} \mathrm{O}_{3} ; \mathrm{CA} 2: 90 \% \mathrm{Al}_{2} \mathrm{O}_{3}$ e $10 \% \mathrm{ZrO}_{2}$; CA-PC: $90 \% \mathrm{Al}_{2} \mathrm{O}_{3}, 5 \% \mathrm{ZrO}_{2}$ e $5 \% \mathrm{SiC}_{\text {whike }}$ ) e três composições à base de LZSA (CF6: $70 \%$ LZSA, $10 \% \mathrm{Al}_{2} \mathrm{O}_{3}$ e $20 \% \mathrm{SiC}_{\text {whisker }}$; CF8: 50\% LZSA, $10 \% \mathrm{Al}_{2} \mathrm{O}_{3}, 20 \% \mathrm{TiO}_{2}$ e $20 \% \mathrm{SiC}_{\text {whisker }}$; CF-PC: $75 \% \mathrm{LZSA}_{2} \% \mathrm{Al}_{2} \mathrm{O}_{3}, 10 \% \mathrm{TiO}_{2}$ e $10 \% \mathrm{SiC}_{\text {whisker }}$ ) foram selecionadas. Estas composições foram projetadas para gerar tensões residuais de compressão nas camadas à base de LZSA (até - 32,35 MPa) e de tração nas camadas à base de $\mathrm{Al}_{2} \mathrm{O}_{3}$ (mínimo de 0,27 MPa). A técnica de tape casting foi empregada para produzir folhas das composições selecionadas, utilizando-se quantidades de ligantes e plastificantes indicadas na literatura. As composições foram então processadas por tape casting, obtendo-se folhas cerâmicas homogêneas, flexíveis e com resistência a verde suficiente para o manuseio. A espessura das folhas das composições de alumina variou de 130 a $210 \mu \mathrm{m}$ e as folhas das composições de LZSA entre 180 e $230 \mu \mathrm{m}$.
\end{abstract}

Palavras-chave: compósito cerâmico multicamada, alumina, vitrocerâmico LZSA, tensões residuais, tape casting .

\begin{abstract}
Ceramic matrix composites have been developed in recent decades as an efficient way of improving the fracture toughness of ceramic materials. Multilayered ceramic composites have been highlighted by their relatively low cost and easy production. These composites are made of stacked layers of two different ceramic materials and present as toughening mechanism the crack path deviation layer by layer in the composite. In this work, compositions based on alumina and $\mathrm{LZSA}$ glass-ceramic $\left(\mathrm{Li}_{2} \mathrm{O}-\mathrm{ZrO}_{2}-\mathrm{SiO}_{2}-\mathrm{Al}_{2} \mathrm{O}_{3}\right)$ were studied to compose the layers in order to obtain multilayer composite with strong interfaces. Thus, compressive residual stresses in thin LZSA-based layers interspersed with $\mathrm{Al}_{2} \mathrm{O}_{3}$-based layers, under low tensile residual stress, were expected to obtain. The compositions were defined by factorial design, generating specimens by pressing that were sintered and characterized by determination of the coefficient of thermal expansion and

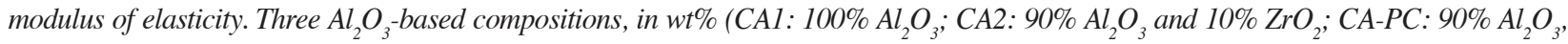
$5 \% \mathrm{ZrO}_{2}$ and $5 \% \mathrm{SiC}_{\text {whisker }}$ ) and three LZSA-based compositions (CF6: 70\% LZSA, $10 \% \mathrm{Al}_{2} \mathrm{O}_{3}$ and 20\% $\mathrm{SiC}_{\text {whisker }} ; \mathrm{CF} 8: 50 \% \mathrm{LZSA}, 10 \%$ $\mathrm{Al}_{2} \mathrm{O}_{3}, 20 \% \mathrm{TiO}_{2}$ and $20 \% \mathrm{SiC}_{\text {whisker }}$; CF-PC: $75 \% \mathrm{LZSA}, 5 \% \mathrm{Al}_{2} \mathrm{O}_{3}, 10 \% \mathrm{TiO}_{2}$ and $10 \% \mathrm{SiC}_{\text {whisker }}$ ) were selected. Such compositions were designed to generate residual compressive stress in the LZSA-based layers (up to -32.35 MPa) and residual tensile stress on the $\mathrm{Al}_{2} \mathrm{O}_{3}$-based layers (at least 0.27 MPa). The tape casting technique was used to produce sheets of the selected compositions, using amounts of binders and plasticizers reported in the literature. The compositions were then prepared by tape casting obtaining homogenous, flexible ceramic sheets showing enough green strength to be handled. The alumina-based sheets thickness ranged between 130 and $210 \mu \mathrm{m}$, while LZSA-based sheets varied between 180 and $230 \mu \mathrm{m}$.
\end{abstract}

Keywords: multilayer ceramic composite, alumina, LZSA glass-ceramic, residual stresses, tape casting.

\section{INTRODUÇÃO}

Materiais cerâmicos encontram diversas aplicações por suas propriedades de alta rigidez, resistência à compressão, resistência ao desgaste, resistência química e habilidade de suportar elevada temperatura. No entanto, sua inerente 
fragilidade e baixas tenacidade e confiabilidade ainda restringem seu uso. A fim de contornar essas deficiências, nas últimas décadas os compósitos de matriz cerâmica têm sido alvo de diversos estudos, considerando diferentes formas de se aperfeiçoar esses materiais. Compósitos de matriz cerâmica têm se mostrado como uma boa opção no que se refere ao aumento da tenacidade, quando comparados ao material monolítico. Neste sentido, os compósitos cerâmicos multicamadas (CCMs), que contam com alternância de camadas de dois cerâmicos distintos, compondo camadas espessas e camadas finas (intercamadas), intercaladas de forma simétrica, destacam-se por seu menor custo de fabricação e mais fácil processamento quando comparados aos compósitos reforçados com fibras, por exemplo [1].

Dois grupos distintos de CCMs são em geral encontrados nos diversos trabalhos publicados sobre o assunto: aqueles que contam com interfaces fortes entre as camadas e aqueles que contam com interfaces fracas. Os detalhes destes dois grupos de CCMs podem ser encontrados em [2]. Importante notar que, em ambos os casos, as camadas componentes do compósito devem ser rigorosamente selecionadas, de modo a se obter o produto final com as características desejadas. No caso específico dos CCMs com interfaces fortes, a escolha de composições para as camadas que sejam capazes de originar as tensões residuais é uma etapa de extrema importância, bem como a definição de suas espessuras, de modo a se maximizar as tensões de compressão (benéficas) e minimizar as tensões de tração (que podem gerar defeitos quando elevadas) [3-5]. Avaliações de características como módulo de elasticidade (E) e coeficiente de expansão térmica (CET) são indispensáveis nesta etapa [6]. Neste sentido, vitrocerâmicos do sistema LZSA $\left(\mathrm{Li}_{2} \mathrm{O}-\mathrm{ZrO}_{2}-\mathrm{SiO}_{2}-\right.$ $\mathrm{Al}_{2} \mathrm{O}_{3}$ ) se destacam devido à sua microestrutura composta de cristais micrométricos de espodumênio- $\beta_{\mathrm{ss}}$ (solução sólida, $\left.\mathrm{Li}_{2} \mathrm{O} \cdot \mathrm{Al}_{2} \mathrm{O}_{3} \cdot 4-10 \mathrm{SiO}_{2}\right)$ e silicato de zircônio $\left(\mathrm{ZrSiO}_{4}\right)$, que conferem a este material densificação à baixa temperatura, baixo CET e valores de propriedades mecânicas que o tornam interessante para emprego, por exemplo, em aplicações de LTCC (low temperature co-fired ceramics) obtidos por tape casting [7-15].

CCMs podem ser fabricados por diferentes processos, que podem gerar as camadas in situ, como slip casting [16] e deposição eletroforética [17], e outras de laminação, nas quais folhas cerâmicas pré-fabricadas são empilhadas e conformadas $[4,18]$. A técnica de tape casting para fabricação destas folhas cerâmicas é uma das mais conhecidas, sendo bastante utilizada por sua versatilidade no emprego de diversas composições e bom acabamento nas peças produzidas. Este estudo objetivou estudar composições adequadas às camadas finas e espessas de um compósito cerâmico multicamada, baseadas em vitrocerâmico do sistema $\mathrm{Li}_{2} \mathrm{O}-\mathrm{ZrO}_{2}-\mathrm{SiO}_{2}-\mathrm{Al}_{2} \mathrm{O}_{3}$ (LZSA) e alumina, respectivamente. A técnica de tape casting aquoso foi empregada na obtenção de folhas de composições avaliadas que demonstraram potencial de utilização em compósitos cerâmicos multicamadas.

\section{MATERIAIS E MÉTODOS}

\section{Materiais utilizados}

Os seguintes materiais cerâmicos foram utilizados para as composições testadas em cada camada: alumina $\left(\mathrm{Al}_{2} \mathrm{O}_{3}-\alpha\right.$, Inframat $^{\circledast}$ Advanced Materials $^{\mathrm{TM}}$, EUA, $99,85 \%$ de pureza) com tamanho médio de partículas, $\mathrm{D}_{50}$, de $350 \mathrm{~nm}$, área de superfície específica de $4,5 \pm 1 \mathrm{~m}^{2} / \mathrm{g}$ e densidade $3,94 \mathrm{~g} / \mathrm{cm}^{3}$; zircônia (TZ-3Y-E, Tosoh, Japão, com 99,9\% de pureza) parcialmente estabilizada com $3 \%$ molar de ítria $\left(\mathrm{Y}_{2} \mathrm{O}_{3}\right)$, de densidade $6,06 \mathrm{~g} / \mathrm{cm}^{3}$ e $\mathrm{D}_{50}$ de $0,6 \mu \mathrm{m}$; whisker de carbeto de silício (SiC- $\beta$ monocristalino, ACM $^{\mathrm{TM}}$ - Advanced Materials Composite, EUA), com diâmetro médio de $0,65 \mu \mathrm{m}$, comprimento médio de 10 a $12 \mu \mathrm{m}$ e densidade de $3,21 \mathrm{~g} / \mathrm{cm}^{3}$; dióxido de titânio $\left(\mathrm{TiO}_{2}\right.$, anatásio, Inframat ${ }^{\circledR}$ Advanced Materials ${ }^{\mathrm{TM}}$, EUA) com tamanho de partícula primária de $40 \mathrm{~nm}$; e frita precursora do vitrocerâmico do sistema LZSA (Tecnofrita Produtos Cerâmicos, Brasil), com diâmetro médio de partícula de 2,23 um, área de superfície

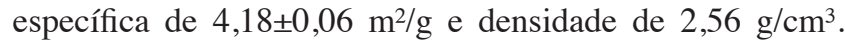
Estes materiais foram selecionados com base nos efeitos esperados nas composições testadas em relação aos objetivos deste trabalho. A alumina é um material cerâmico muito utilizado para obtenção de artefatos de elevado desempenho mecânico [3, 4, 6, 16, 18], assim como a zircônia estabilizada com ítria $[3,4,16,18]$. Whiskers de $\mathrm{SiC}\left(\mathrm{SiC}_{\mathrm{w}}\right)$ têm sido empregados como material de reforço em compósitos à base de alumina e outros [5]. Vitrocerâmico do sistema LZSA tem sido empregado na obtenção de compósitos, em especial à base de alumina, para auxiliar na sinterização, nas propriedades mecânicas e na redução do CET [11, 1315], enquanto que o $\mathrm{TiO}_{2}$ tem sido empregado neste sistema vitrocerâmico como agente nucleante e para reduzir o CET $[19,20]$. Os aditivos utilizados foram: álcool isopropílico P.A. (Vetec Química Fina, Brasil) como solvente auxiliar; poliacrilato de amônio Roltan AP-700 (Khemeia, Brasil) como dispersante; polietilenoglicol (PEG) PA 400 (Synth, Brasil) como plastificante; e Optapix PA 42 [ZschimmerSchwarz, Alemanha; granulado à base de poli(álcool vinílico)], Trucel 10 (Zschimmer-Schwarz, Alemanha; à base de carboximetilcelulose de baixa viscosidade) e Mowilith LDM 6138 (Archroma, à base de resinas acrílicas) como ligantes. Os ligantes Optapix e Trucel, originalmente na forma de grânulos, foram dissolvidos em água deionizada, sob agitação a $80^{\circ} \mathrm{C}$, para formar soluções de, respectivamente, 20 e $10 \%$ em massa.

\section{Procedimento experimental}

O procedimento experimental adotado foi dividido em duas etapas: estudo das tensões residuais teóricas e obtenção das folhas cerâmicas. Para se estudar as composições e avaliar a adequabilidade destas para a formação dos compósitos, um conjunto de diferentes composições definidas por planejamento experimental fatorial com três pontos centrais foi proposto, conforme demonstrado nas 
Tabela I - Estrutura do planejamento fatorial $2^{2}$ com três pontos centrais para a camada de tração ( $\%$ em massa).

[Table I - Factorial design $2^{2}$ with three central points for the tensile layer $(w t \%)$.]

\begin{tabular}{ccccc}
\hline Ensaio & Amostra & $\mathrm{Al}_{2} \mathrm{O}_{3}$ & $\mathrm{ZrO}_{2}$ & $\mathrm{SiC}_{\mathrm{w}}$ \\
\hline 1 & $\mathrm{CA} 1$ & 100 & 0 & 0 \\
2 & $\mathrm{CA} 2$ & 90 & 10 & 0 \\
3 & $\mathrm{CA} 3$ & 90 & 0 & 10 \\
4 & $\mathrm{CA} 4$ & 80 & 10 & 10 \\
$5,6,7$ & CA-PC (CA5, CA6, CA7) & 90 & 5 & 5 \\
\hline
\end{tabular}

Tabelas I e II. Corpos de prova dessas composições foram obtidos via prensagem uniaxial e queima em forno elétrico de laboratório. A preparação das composições foi feita por moagem em moinho de alta energia (Retzsch, Alemanha), utilizando-se jarro de alumina de $500 \mathrm{~mL}$ e esferas de alumina ( $5 \mathrm{~mm}$ de diâmetro), em moagem a $200 \mathrm{rpm}$ por $1 \mathrm{~h}$, em lotes de $220 \mathrm{~g}$ de suspensão aquosa $(50 \%$ de sólidos). Os pós à base de alumina (composições da Tabela I) foram preparados com $1 \%$ de plastificante (PEG) e $1 \%$ de ligante (Optapix) sobre a massa de sólido. Os pós à base de LZSA foram preparados com 5\% de ligante (Trucel) e $5 \%$ de plastificante (PEG). As suspensões foram secadas em estufa com circulação de ar até massa constante e granuladas manualmente com almofariz e pistilo de ágata, até se obter grânulos passantes em peneira de abertura $500 \mu \mathrm{m}$. Peças de $12 \mathrm{~cm}$ x $6,25 \mathrm{~cm} \times 0,5 \mathrm{~cm}$ foram obtidas por prensagem, utilizando-se $100 \mathrm{~g}$ de pó e $110 \mathrm{MPa}$ para as composições de alumina e $80 \mathrm{~g}$ e $100 \mathrm{MPa}$ para aquelas de LZSA. Estas peças foram então cortadas para se ajustar as dimensões aos ensaios mecânicos, com o auxílio de uma navalha, e lixadas para se obter corpos de prova de $6,25 \mathrm{~cm} \times 1,5 \mathrm{~cm}$, os quais foram queimados. As temperaturas e os tempos de queima foram determinados em dilatômetro óptico (Misura HSM ODHT, Itália). O ensaio foi realizado da seguinte forma: para os corpos de prova de LZSA ( $3 \mathrm{~mm}$ de altura e $2 \mathrm{~mm}$ de diâmetro), taxa de $40{ }^{\circ} \mathrm{C} / \mathrm{min}$ até a temperatura de fusão e resfriamento natural dentro do forno; para os corpos de prova das composições de alumina ( $5 \mathrm{~mm} \times 5 \mathrm{~mm} \times 12 \mathrm{~mm}$ ), $1{ }^{\circ} \mathrm{C} /$ min até $500{ }^{\circ} \mathrm{C}, 5^{\circ} \mathrm{C} / \mathrm{min}$ até $1600{ }^{\circ} \mathrm{C}$ e patamar de 10 $\mathrm{h}$, com resfriamento natural dentro do forno. Os corpos de prova foram queimados de acordo com os ciclos térmicos definidos na dilatometria óptica e testados quanto a E e CET. $\mathrm{O}$ módulo de elasticidade (E) foi medido em um analisador de vibrações transitórias (ATCP Sonelastic, Brasil; $50 \mathrm{~mm}$ x $10 \mathrm{~mm}$ x $3 \mathrm{~mm}$ ). O CET foi determinado em dilatômetro de contato (Netzsch, DIL 402 C, Alemanha; $25 \mathrm{~mm}$ x $3 \mathrm{~mm}$ x $3 \mathrm{~mm}$ ).

Estes dados foram então combinados, assumindo as composições de LZSA como componente da camada de compressão e as composições de alumina como camadas de tração, nas equações referidas em [4], para se determinar quais composições se adequariam ao compósito proposto e qual a intensidade prevista para as tensões residuais. Estes compósitos apresentaram número de camadas ímpar, uma vez que as camadas externas (topo e base) devem ser iguais. Assim, as camadas que incluíram a parte externa foram designadas pelo índice 1 (camada sob tração) e as camadas internas pelo índice 2 (camada sob compressão). O número de camadas designado pelo índice 1 foi $(\mathrm{N}+1) / 2$ e pelo índice 2 foi (N-1)/2 [4]. Desta forma, as tensões residuais nas camadas de tração e de compressão foram representadas por:

$$
\begin{gathered}
\sigma_{r 1}=\frac{E_{1}{ }^{\prime} E_{2}^{\prime} f_{2}\left(\alpha_{T 2}-\alpha_{T 1}\right) \Delta T}{E_{1}{ }^{\prime} f_{1}+E_{2} f_{2}{ }^{\prime}} \\
\sigma_{r 2}=\frac{E_{2}{ }^{\prime} E_{1}^{\prime} f_{1}\left(\alpha_{T 1}-\alpha_{T 2}\right) \Delta T}{E_{1}{ }^{\prime} f_{1}+E_{2} f_{2}{ }^{\prime}}
\end{gathered}
$$

onde, $\quad E_{j}^{\prime}=E /\left(1-v_{j}\right), \quad f_{l}=(N+1) l_{1} / 2 w, \quad f_{2}=(N-1) l_{2} / 2 w, \quad E_{j}$ é o módulo de elasticidade e $v_{j}$ é a razão de Poisson do componente $j, l_{1}$ e $l_{2}$ são as espessuras das camadas do primeiro e segundo componente, $\alpha_{T 1}$ e $\alpha_{T 2}$ são os coeficientes de expansão térmica (CET) do primeiro e

Tabela II - Estrutura do planejamento fatorial $2^{3}$ com três pontos centrais para a camada de compressão (à base de LZSA; \% em massa).

[Table II - Factorial design $2^{3}$ with three central points for the compressive layer (based on LZSA; wt\%).]

\begin{tabular}{cccccc}
\hline Ensaio & Amostra & LZSA & $\mathrm{Al}_{2} \mathrm{O}_{3}$ & $\mathrm{TiO}_{2}$ & $\mathrm{SiC}_{\mathrm{w}}$ \\
\hline 1 & $\mathrm{CF} 1$ & 100 & 0 & 0 & 0 \\
2 & $\mathrm{CF} 2$ & 90 & 10 & 0 & 0 \\
3 & $\mathrm{CF} 3$ & 80 & 0 & 20 & 0 \\
4 & $\mathrm{CF} 4$ & 70 & 10 & 20 & 0 \\
5 & $\mathrm{CF} 5$ & 80 & 0 & 0 & 20 \\
6 & $\mathrm{CF} 6$ & 70 & 10 & 0 & 20 \\
7 & $\mathrm{CF} 7$ & 60 & 0 & 20 & 20 \\
8 & $\mathrm{CF} 8$ & 50 & 10 & 20 & 20 \\
$9,10,11$ & $\mathrm{CF}-\mathrm{PC}(\mathrm{CF} 9, \mathrm{CF} 10, \mathrm{CF} 11)$ & 75 & 5 & 10 & 10 \\
\hline
\end{tabular}




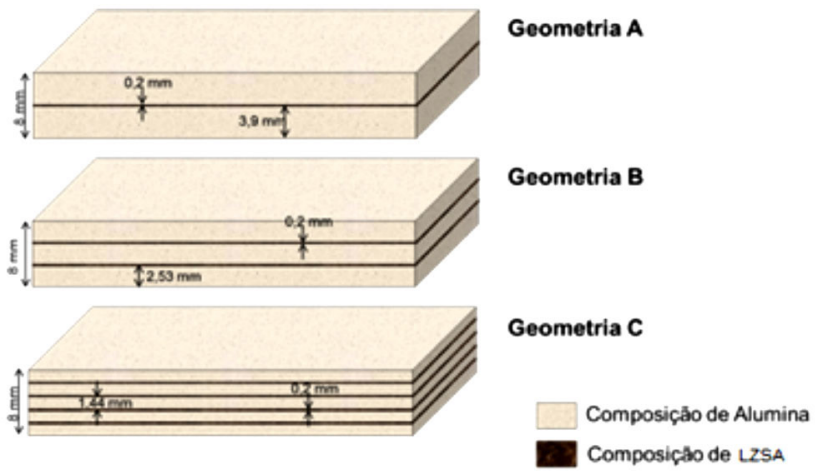

Figura 1: Geometrias propostas indicando as espessuras das camadas.

[Figure 1: Proposed configurations indicating the thickness of the layers.]

segundo componente, respectivamente, $\Delta T$ é a diferença entre a temperatura de acoplamento (temperatura na qual as camadas são livres de tensões) e a temperatura atual, e $w$ é a espessura total do espécime [4]. As geometrias consideradas para os cálculos são mostradas na Fig. 1 .

Escolhidas as composições adequadas, foram então preparadas as folhas cerâmicas pela técnica de tape casting. Para tal, um breve estudo foi realizado para se determinar as quantidades de dispersante que deveriam ser adicionadas em cada composição, através de teste para determinação do tempo de escoamento em copo Ford de $100 \mathrm{~mL}$, variando-se a quantidade de dispersante entre 0 e $1,0 \%$ sobre a massa de sólido e mantendo-se inalteradas as quantidades de solvente, ligante e dispersante nas suspensões, já previamente estabelecidas via observação visual da qualidade das folhas. Assim, chegou-se nas composições para a produção das folhas cerâmicas apresentadas na Tabela III.

As suspensões para o tape casting foram homogeneizadas em moinho de alta energia, a $200 \mathrm{rpm}$ por $1 \mathrm{~h}$. As suspensões das composições baseadas em LZSA foram aplicadas imediatamente após a homogeneização sobre folhas de

Tabela III - Composições adotadas (\% em massa) das suspensões para técnica de colagem de folha cerâmica.

[Table III - Compositions (wt\%) of the suspensions for the tape casting.]

\begin{tabular}{|c|c|c|c|c|}
\hline Componente & \multicolumn{2}{|c|}{$\begin{array}{c}\text { Suspensão de } \\
\text { LZSA }\end{array}$} & \multicolumn{2}{|c|}{$\begin{array}{l}\text { Suspensão de } \\
\text { alumina }\end{array}$} \\
\hline Solvente & $\begin{array}{c}\text { Água } \\
\text { deionizada } \\
\text { Álcool } \\
\text { isopropílico }\end{array}$ & $\begin{array}{l}19,0 \\
15,0\end{array}$ & $\begin{array}{c}\text { Água } \\
\text { deionizada }\end{array}$ & 29,5 \\
\hline Dispersante & $\begin{array}{l}\text { Roltan } \\
\text { AP-700 }\end{array}$ & 1,0 & $\begin{array}{c}\text { Roltan AP- } \\
700\end{array}$ & 0,5 \\
\hline Pó cerâmico & $\begin{array}{c}\text { Composição } \\
\text { de LZSA }\end{array}$ & 40,0 & $\begin{array}{c}\text { Composição } \\
\text { de alumina }\end{array}$ & 50,0 \\
\hline Plastificante & PEG & 5,0 & PEG & 5,0 \\
\hline Ligante & Mowilith & 20,0 & Optapix & 15,0 \\
\hline
\end{tabular}

poliestirenotereftalato. As suspensões das composições baseadas em alumina foram aplicadas sobre papel alumínio após descanso por $24 \mathrm{~h}$ para eliminação de bolhas de ar provenientes da etapa de homogeneização. A aplicação foi realizada manualmente, utilizando-se um binil com abertura de $0,5 \mathrm{~mm}$ e velocidade de aplicação de cerca de $0,15 \mathrm{~m} / \mathrm{s}$. Após o tempo de descanso, os tapes das composições a base de LZSA e de alumina foram retirados das folhas de poliestirenotereftalato e de alumínio, respectivamente. Desta forma, folhas (tapes) de cada composição foram obtidas individualmente e que poderiam ser consolidadas em estruturas multicamadas, de acordo com as configurações mostradas na Fig. 1. Estudo posterior será realizado para sinterizar tais estruturas multicamadas e caracterizá-las.

A densidade relativa $\left(\varrho_{\text {relativa }}\right)$ dos corpos de prova sinterizados foi calculada a partir da relação entre a densidade aparente e a densidade teórica. A densidade aparente $\left(\varrho_{\text {queimado }}\right)$ foi determinada por imersão em água $\left(22 \pm 1{ }^{\circ} \mathrm{C}\right)$. A densidade teórica $\left(\varrho_{\text {teórica }}\right)$ foi medida por picnometria a hélio (Quantachrome Ultrapyc 1200e, EUA). A tenacidade à fratura $\left(\mathrm{K}_{\mathrm{Ic}}\right)$ foi determinada pelo método do entalhe (single edge notched beam - SENB), com profundidade do entalhe de $40 \%$ da espessura total da amostra. Em seguida, os corpos de prova foram submetidos ao ensaio de resistência à flexão pelo método a três pontos em uma máquina universal de ensaios mecânicos (EMIC DL10000, Brasil). O K $\mathrm{Ic}_{\mathrm{Ic}}$ do material foi calculado pela equação de Griffith:

$$
\mathrm{K}_{\mathrm{Ic}}=\sigma \cdot \mathrm{Y} \cdot \sqrt{\mathrm{a}}=\sqrt{\mathrm{s} \cdot \mathrm{E} \cdot \mathrm{y}_{\mathrm{i}}}
$$

onde, $\mathrm{K}_{\mathrm{Ic}}$ é a tenacidade à fratura $\left(\mathrm{MPa} \cdot \mathrm{m}^{0,5}\right)$, $\sigma$ é a tensão máxima de ruptura (MPa), E é o módulo de elasticidade $(\mathrm{MPa}), \gamma_{i}$ é a energia de fratura $\left(\mathrm{J} / \mathrm{m}^{2}\right)$, Y é o fator de forma e $a$ é a profundidade do entalhe ( $\mathrm{mm})$. O fator de forma para este tipo de entalhe é dado por:

$Y=1,99-2,47\left(\frac{a}{B}\right)+12,97\left(\frac{a}{B}\right)^{2}-23,17\left(\frac{a}{B}\right)^{3}+24,8\left(\frac{a}{B}\right)^{4}$

onde, $B$ é a largura do corpo de prova ( $\mathrm{mm})$.

\section{RESULTADOS E DISCUSSÃO}

\section{Caracterização das amostras}

Os resultados obtidos na caracterização das amostras monolíticas são mostrados nas Tabelas IV e V. A densificação das amostras de composições de alumina (Tabela IV) foi dificultada pela presença de whiskers de $\mathrm{SiC}$, enquanto que a zircônia, como era de se esperar, melhorou a densificação. Como não foi realizado controle de atmosfera no forno, é possível que tenha havido reação de oxidação do $\mathrm{SiC}$, gerando porosidade excessiva nos corpos de prova. A porosidade afeta especialmente o valor de E dos corpos de prova destas composições, nas quais também se observou diminuição do valor da tenacidade à fratura $\left(\mathrm{K}_{\mathrm{Ic}}\right)$. As composições à base de LZSA não ficaram bem densificadas no processo de queima (Tabela V), apesar dos 
Tabela IV - Propriedades térmicas e mecânicas das composições à base de alumina sinterizadas a $1600^{\circ} \mathrm{C}$ por $10 \mathrm{~h}$. [Table IV - Thermal and mechanical properties of the alumina-based compositions sintered at $1600{ }^{\circ} \mathrm{C}$ for $10 \mathrm{~h}$.]

\begin{tabular}{cccccccc}
\hline Propriedade & CA1 & CA2 & CA3 & CA4 & CA5 & CA6 & CA7 \\
\hline$\varrho_{\text {seco }}\left(\mathrm{g} / \mathrm{cm}^{3}\right)$ & 2,50 & 2,59 & 2,28 & 2,33 & 2,40 & 2,38 & 2,41 \\
$\varrho_{\text {queimado }}\left(\mathrm{g} / \mathrm{cm}^{3}\right)$ & 3,79 & 4,00 & 2,71 & 3,30 & 3,63 & 3,60 & 3,63 \\
$\varrho_{\text {teórica }}\left(\mathrm{g} / \mathrm{cm}^{3}\right)$ & 3,94 & 4,15 & 3,87 & 4,08 & 4,01 & 4,01 & 4,01 \\
$\varrho_{\text {relativa }}(\%)$ & 96,16 & 96,33 & 70,02 & 80,86 & 90,53 & 89,85 & 90,51 \\
$\mathrm{E}(\mathrm{GPa})$ & 360,6 & 359,1 & 130,9 & 233,0 & 283,2 & 265,4 & 284,9 \\
$\mathrm{CET}\left(10^{-6}{ }^{\circ} \mathrm{C}^{-1}\right)$ & 7,58 & 7,75 & 5,92 & 6,01 & 6,86 & 6,86 & 6,81 \\
$\mathrm{~K}_{\text {Ic }}\left(\mathrm{MPa} \cdot \mathrm{m}^{1 / 2}\right)$ & 3,74 & 3,75 & 2,06 & 2,61 & 2,75 & 3,13 & 3,04 \\
\hline
\end{tabular}

Tabela V - Propriedades térmicas e mecânicas das composições à base de LZSA sinterizadas a $770{ }^{\circ} \mathrm{C}\left(\mathrm{CF} 1\right.$ a CF4) ou $900{ }^{\circ} \mathrm{C}$ (CF5 a CF11), com resfriamento controlado.

[Table V - Thermal and mechanical properties of LZSA-based compositions sintered at $770{ }^{\circ} \mathrm{C}$ (CF1 to CF4) or $900{ }^{\circ} \mathrm{C}$ (CF5 to CF11) with controlled cooling.]

\begin{tabular}{cccccccccccc}
\hline Propriedade & CF1 & CF2 & CF3 & CF4 & CF5 & CF6 & CF7 & CF8 & CF9 & CF10 & CF11 \\
\hline$\varrho_{\text {seco }}\left(\mathrm{g} / \mathrm{cm}^{3}\right)$ & 1,52 & 1,67 & 1,74 & 1,80 & 1,45 & 1,56 & 1,70 & 1,80 & 1,63 & 1,70 & 1,71 \\
$\varrho_{\text {queimado }}\left(\mathrm{g} / \mathrm{cm}^{3}\right)$ & 1,12 & 1,51 & 1,69 & 2,03 & 1,36 & 1,43 & 1,61 & 1,75 & 1,72 & 1,65 & 1,74 \\
$\varrho_{\text {teórica }}\left(\mathrm{g} / \mathrm{cm}^{3}\right)$ & 2,56 & 2,70 & 2,89 & 3,03 & 2,69 & 2,83 & 3,94 & 3,16 & 2,86 & 2,86 & 2,86 \\
$\varrho_{\text {relativa }}(\%)$ & 43,78 & 56,00 & 58,27 & 66,93 & 50,49 & 53,11 & 53,11 & 55,32 & 60,01 & 57,68 & 60,71 \\
$\mathrm{E}(\mathrm{GPa})$ & 25,57 & 32,10 & 41,19 & 53,36 & 24,45 & 19,05 & 19,58 & 21,51 & 23,67 & 21,93 & 21,65 \\
$\mathrm{CET}\left(10^{-6}{ }^{\circ} \mathrm{C}^{-1}\right)$ & 10,21 & 9,41 & 9,57 & 8,62 & 8,30 & 7,06 & 9,11 & 6,24 & 6,39 & 6,01 & 6,35 \\
\hline
\end{tabular}

cuidados adotados. A porosidade excessiva, que chegou, em alguns casos, a mais de $50 \%$, prejudicou as características mecânicas destes materiais e pode ter influenciado no valor do CET, desviando estes valores do esperado segundo dados abstraídos de [8], que obteve o valor de $4,9 \times 10^{-6}{ }^{\circ} \mathrm{C}^{-1}$ para este sistema vitrocerâmico.

A geração de tensões residuais é dependente principalmente da diferença de CET entre as amostras que compõem as camadas. No compósito em estudo, as camadas de alumina, conforme proposto, devem estar sob tração e, portanto, devem apresentar CET superior às camadas de compressão. De acordo com os valores obtidos, verificou-se que somente as composições CA1, CA2 e o ponto central (CA5, CA6 e CA7, ora em diante CA-PC), entre as composições à base de alumina, apresentaram CET superior a algumas das composições à base de LZSA. Entre as camadas de LZSA, as composições CF6, CF8 e o ponto central (CF9, CF10 e CF11, ora em diante CFPC) apresentaram CET inferior àqueles das composições de alumina; portanto, foram as mais adequadas a gerar as tensões residuais de compressão.

\section{Estudo das tensões residuais teóricas}

Temperatura de acoplamento e temperatura de sinterização: a temperatura de acoplamento, ou seja, temperatura a partir da qual no resfriamento as tensões residuais devem se estabelecer, no caso estudado, deve ser determinada pela camada de LZSA, uma vez que esta sinteriza e amolece em temperatura muito abaixo da temperatura de sinterização da alumina ou de suas composições. Ensaios de dilatometria óptica das composições de alumina e de LZSA confirmaram este comportamento, como pode ser visto nas Figs. 2 e 3. Conforme esperado, expansão ocorreu nas composições CF6, CF8 e CF-PC (Fig. 2) devido à presença de $\mathrm{SiC}$, já que a atmosfera do forno não foi redutora. Entretanto, a composição CF8, contendo $20 \%$ de $\mathrm{TiO}_{2}$, apresentou menor expansão térmica. Desta forma, compreendeu-se que a temperatura de acoplamento dependeu das camadas de compressão (LZSA), enquanto que a temperatura de sinterização dos compósitos foi determinada pelas camadas de tração (alumina). Assumiu-se como temperatura de acoplamento a temperatura referente ao último pico de cristalização de LZSA, ou seja, $815^{\circ} \mathrm{C}$. Esta temperatura está situada próximo às temperaturas de amolecimento das amostras (Fig. 2), e é mais conservadora do que assumir a temperatura de transição vítrea do LZSA, uma vez que, cristalizando-se, o vitrocerâmico já poderia gerar tensões, que seriam subestimadas se fosse utilizada a temperatura de referência muito inferior no equacionamento.

A temperatura de sinterização do compósito formado pelo sistema alumina/LZSA, por sua vez, deve ser igual ou superior a $1600{ }^{\circ} \mathrm{C}$. Conforme Fig. 3, nesta temperatura ainda não se observou a densificação máxima; portanto, temperaturas inferiores seriam ineficazes. No entanto, o aumento do patamar de sinterização interfere na densificação 


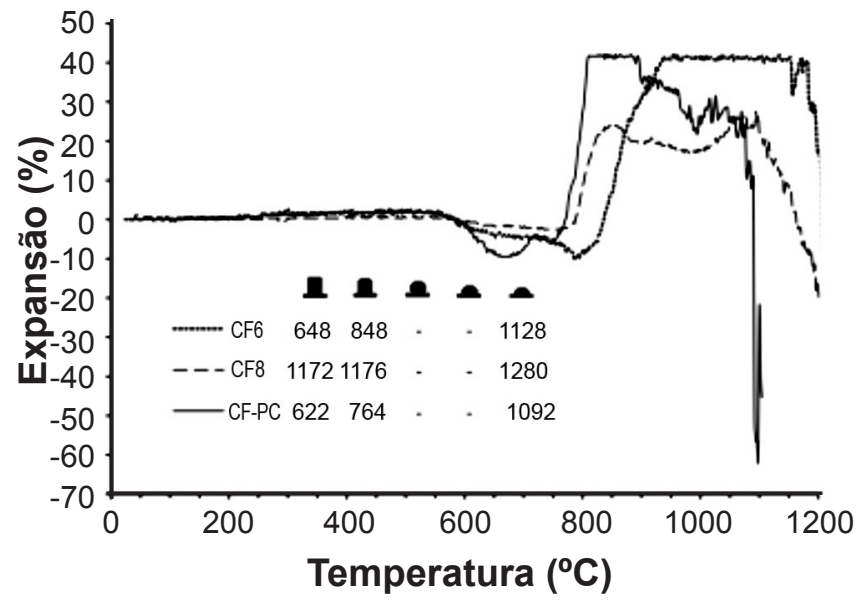

Figura 2: Ensaio de dilatometria óptica das composições de LZSA selecionadas para as camadas de compressão. $\mathbf{I}$ é a temperatura de sinterização, $\mathbf{\ell}$ é a temperatura de amolecimento, e é a temperatura de esfera, $\mathbf{e}$ é a temperatura de meia esfera e $\mathbf{e}$ é a temperatura de fusão, dadas em ${ }^{\circ} \mathrm{C}$.

[Figure 2: Results of optical dilatometry of the selected LZSAbased compositions for the compressive layers. $\mathbf{n}$ is the sintering temperature, $\mathbf{n}$ is the softening temperature, $\mathbf{e}$ is the sphere temperature, $\mathbf{2}$ is the half sphere temperature, and $\mathbf{n}$ is the melting temperature, given in ${ }^{\circ} \mathrm{C}$.]

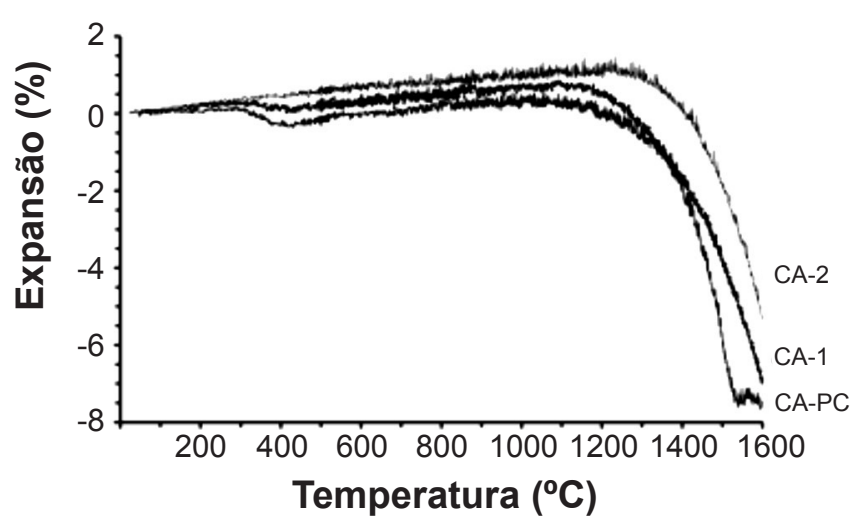

Figura 3: Resultados de dilatometria óptica das composições de alumina selecionadas para as camadas de tração.

[Figure 3: Results of optical dilatometry of the selected aluminabased compositions for the tensile layers.]

do material. De fato, a Fig. 4 mostra que a retração térmica (RT) das composições à base de alumina é fortemente afetada pelo tempo (patamar); entretanto, já não retraem (sinterizam) significativamente a partir de $10 \mathrm{~h}$ de patamar a $1600{ }^{\circ} \mathrm{C}$. Os corpos de prova foram confeccionados com empilhamento de folhas das composições de alumina, a fim de verificar mais fidedignamente o comportamento esperado para a sinterização de compósitos feitos a partir desta técnica. Os corpos de prova sinterizados neste ensaio apresentaram densidade aparente de $3,68 \mathrm{~g} / \mathrm{cm}^{3}$ para CA1 (93,4\% da densidade teórica), $3,80 \mathrm{~g} / \mathrm{cm}^{3}$ para CA2 $(91,5 \%)$ e $3,54 \mathrm{~g} / \mathrm{cm}^{3}(88,3 \%)$ para a CA-PC. Estes dados, somados à tendência de densificação ainda perceptível nos gráficos, demostraram que um patamar superior ao tempo de $10 \mathrm{~h}$ poderia ser aplicado, se o objetivo fosse obter a máxima densificação. Entretanto, limitou-se o patamar de $10 \mathrm{~h}$ para se evitar o crescimento acentuado de grãos de alumina. De fato, é conhecido que o crescimento de grão acentuado da alumina tem forte interferência (redução) em suas propriedades mecânicas [21, 22].

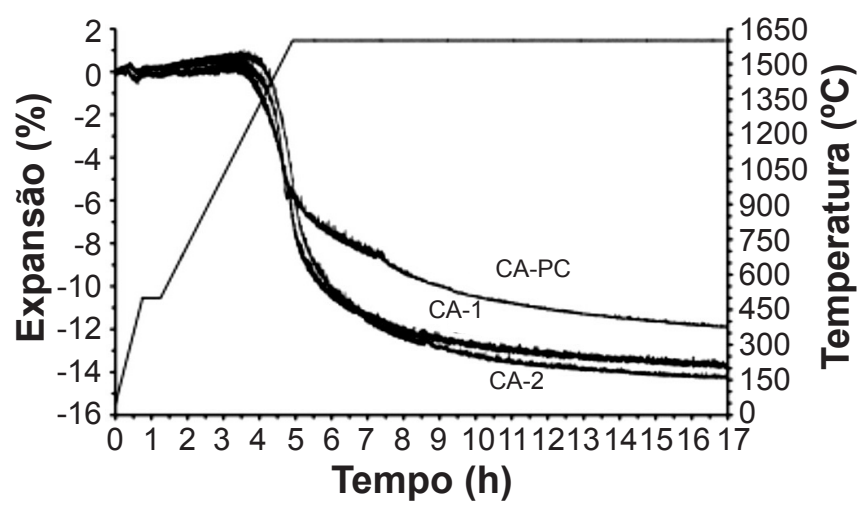

Figura 4: Comportamento de sinterização das composições de alumina feitas com folhas cerâmicas das composições selecionadas, com temperatura máxima de $1600{ }^{\circ} \mathrm{C}$ e patamar de $10 \mathrm{~h}$.

[Figure 4: Sintering behavior of the alumina-based compositions obtained from the selected ceramic tapes and maximum temperature of $1600^{\circ} \mathrm{C}$ and $10 \mathrm{~h}$ holding time.]

Cálculo das tensões: inserindo-se os dados de E e CET, bem como os dados referentes à geometria dos compósitos, nas Equações A e B, pôde-se obter os dados de tensões residuais que devem ser estabelecidos a partir do resfriamento. A razão de Poisson foi assumida ser de 0,25 para todos os casos. A Tabela VI apresenta as tensões residuais teóricas previstas para todas as combinações possíveis entre as composições à base de LZSA e de alumina, para as três geometrias propostas. Estas combinações apresentaram potencialidade de originar tensões compressivas (indicadas pelo sinal negativo) nas camadas com composições à base de LZSA e tensões baixas de tração nas camadas com composição à base de alumina, o que é muito promissor no desenvolvimento de propriedades mecânicas aprimoradas para este sistema. Percebeu-se que as tensões residuais de compressão foram maiores na geometria $\mathrm{A}$, na qual a razão entre as espessuras foi máxima, com apenas uma camada de compressão inserida entre duas espessas camadas de tração. Uma leve diminuição das tensões residuais de compressão foi verificada ao se elevar o número de camadas (geometrias $\mathrm{B}$ e C); no entanto, a diferença foi pequena e não deve comprometer as características finais, mas sim contribuir com maior energia de fratura, visto que há maiores possibilidades de desvio de trincas em propagação pela presença de camadas compressivas no interior do compósito. O projeto de produção de compósitos cerâmicos multicamadas, portanto, incluiria as três geometrias propostas, com combinação entre três composições à base de alumina e três composições à base de LZSA. Desta forma, vinte e quatro compósitos diferentes poderiam ser obtidos para avaliação das propriedades mecânicas. 
Tabela VI - Tensões residuais teóricas para as diversas combinações possíveis entre as combinações de camadas alumina/ LZSA. Em destaque as combinações com potencialidade de originar as tensões adequadas.

[Table VI - Theoretical residual stresses for the different set of possible configurations among the alumina/LZSA layers. Configuration sets with potentiality of generating suitable residual stresses are highlighted.]

\begin{tabular}{|c|c|c|c|c|c|c|c|}
\hline \multirow{2}{*}{ Componente 1} & \multirow{2}{*}{ Componente 2} & \multicolumn{2}{|c|}{ Geometria A } & \multicolumn{2}{|c|}{ Geometria B } & \multicolumn{2}{|c|}{ Geometria C } \\
\hline & & $\sigma_{1}(\mathrm{MPa})$ & $\sigma_{2}(\mathrm{MPa})$ & $\sigma_{1}(\mathrm{MPa})$ & $\sigma_{2}(\mathrm{MPa})$ & $\sigma_{1}(\mathrm{MPa})$ & $\sigma_{2}(\mathrm{MPa})$ \\
\hline CA1 & CF1 & $-1,87$ & 72,94 & $-3,83$ & 72,81 & $-8,06$ & 72,51 \\
\hline CA1 & $\mathrm{CF} 2$ & $-1,63$ & 63,69 & $-3,34$ & 63,54 & $-7,02$ & 63,21 \\
\hline CA1 & $\mathrm{CF} 3$ & $-2,28$ & 88,81 & $-4,66$ & 88,54 & $-9,77$ & 87,96 \\
\hline CA1 & CF4 & $-1,54$ & 60,08 & $-3,15$ & 59,84 & $-6,59$ & 59,33 \\
\hline CA1 & CF5 & $-0,49$ & 19,10 & $-1,00$ & 19,06 & $-2,11$ & 18,99 \\
\hline CA1 & CF6 & 0,28 & $-10,75$ & 0,56 & $-10,73$ & 1,19 & $-10,70$ \\
\hline CA1 & CF7 & $-0,83$ & 32,51 & $-1,71$ & 32,46 & $-3,60$ & 32,36 \\
\hline CA1 & CF8 & 0,80 & $-31,27$ & 1,64 & $-31,22$ & 3,46 & $-31,12$ \\
\hline CA1 & CF-PC & 0,83 & $-32,35$ & 1,70 & $-32,29$ & 3,58 & $-32,18$ \\
\hline CA2 & CF1 & $-1,31$ & 51,20 & $-2,69$ & 51,12 & $-5,66$ & 50,96 \\
\hline $\mathrm{CA} 2$ & $\mathrm{CF} 2$ & $-1,11$ & 43,35 & $-2,28$ & 43,28 & $-4,79$ & 43,11 \\
\hline CA2 & $\mathrm{CF} 3$ & $-1,56$ & 60,96 & $-3,20$ & 60,82 & $-6,72$ & 60,52 \\
\hline CA2 & $\mathrm{CF} 4$ & $-0,97$ & 37,73 & $-1,98$ & 37,61 & $-4,15$ & 37,37 \\
\hline CA2 & CF5 & $-0,28$ & 10,95 & $-0,58$ & 10,93 & $-1,21$ & 10,90 \\
\hline CA2 & CF6 & 0,27 & $-10,70$ & 0,56 & $-10,69$ & 1,19 & $-10,67$ \\
\hline CA2 & CF7 & $-0,56$ & 21,68 & $-1,14$ & 21,66 & $-2,40$ & 21,60 \\
\hline CA2 & CF8 & 0,68 & $-26,44$ & 1,39 & $-26,41$ & 2,93 & $-26,34$ \\
\hline CA2 & CF-PC & 0,70 & $-27,37$ & 1,44 & $-27,34$ & 3,03 & $-27,26$ \\
\hline CA3 & CF1 & $-2,28$ & 89,07 & $-4,67$ & 88,72 & $-9,77$ & 87,97 \\
\hline CA3 & $\mathrm{CF} 2$ & $-2,33$ & 90,88 & $-4,76$ & 90,43 & $-9,94$ & 89,48 \\
\hline CA3 & $\mathrm{CF} 3$ & $-3,12$ & 121,79 & $-6,37$ & 121,03 & $-13,27$ & 119,40 \\
\hline CA3 & CF4 & $-2,99$ & 116,51 & $-6,08$ & 115,56 & $-12,62$ & 113,56 \\
\hline CA3 & CF5 & $-1,21$ & 47,26 & $-2,48$ & 47,08 & $-5,19$ & 46,70 \\
\hline CA3 & CF6 & $-0,45$ & 17,65 & $-0,93$ & 17,60 & $-1,94$ & 17,49 \\
\hline CA3 & CF7 & $-1,30$ & 50,76 & $-2,66$ & 50,61 & $-5,59$ & 50,28 \\
\hline CA3 & CF8 & $-0,14$ & 5,59 & $-0,29$ & 5,57 & $-0,61$ & 5,53 \\
\hline CA3 & CF-PC & $-0,15$ & 6,01 & $-0,32$ & 5,99 & $-0,66$ & 5,94 \\
\hline CA4 & CF1 & $-2,24$ & 87,34 & $-4,59$ & 87,15 & $-9,64$ & 86,73 \\
\hline CA4 & $\mathrm{CF} 2$ & $-2,27$ & 88,71 & $-4,66$ & 88,47 & $-9,77$ & 87,94 \\
\hline CA4 & $\mathrm{CF} 3$ & $-3,05$ & 119,10 & $-6,25$ & 118,68 & $-13,09$ & 117,77 \\
\hline CA4 & $\mathrm{CF} 4$ & $-2,90$ & 113,01 & $-5,92$ & 112,49 & $-12,38$ & 111,38 \\
\hline CA4 & CF5 & $-1,17$ & 45,54 & $-2,39$ & 45,44 & $-5,03$ & 45,24 \\
\hline CA4 & CF6 & $-0,42$ & 16,28 & $-0,86$ & 16,25 & $-1,80$ & 16,19 \\
\hline CA4 & CF7 & $-1,27$ & 49,39 & $-2,60$ & 49,31 & $-5,46$ & 49,12 \\
\hline CA4 & CF8 & $-0,10$ & 4,02 & $-0,21$ & 4,02 & $-0,44$ & 4,00 \\
\hline CA4 & CF-PC & $-0,11$ & 4,38 & $-0,23$ & 4,37 & $-0,48$ & 4,35 \\
\hline CA-PC & CF1 & $-1,80$ & 70,04 & $-3,68$ & 69,91 & $-7,74$ & 69,63 \\
\hline CA-PC & $\mathrm{CF} 2$ & $-1,72$ & 67,00 & $-3,52$ & 66,84 & $-7,39$ & 66,51 \\
\hline CA-PC & $\mathrm{CF} 3$ & $-2,34$ & 91,27 & $-4,79$ & 91,00 & $-10,05$ & 90,42 \\
\hline CA-PC & $\mathrm{CF} 4$ & $-1,97$ & 76,98 & $-4,04$ & 76,68 & $-8,45$ & 76,05 \\
\hline CA-PC & CF5 & $-0,74$ & 28,98 & $-1,52$ & 28,93 & $-3,20$ & 28,82 \\
\hline CA-PC & CF6 & $-0,09$ & 3,36 & $-0,18$ & 3,35 & $-0,37$ & 3,34 \\
\hline CA-PC & CF7 & $-0,93$ & 36,12 & $-1,90$ & 36,07 & $-4,00$ & 35,96 \\
\hline CA-PC & CF8 & 0,27 & $-10,56$ & 0,55 & $-10,54$ & 1,17 & $-10,51$ \\
\hline CA-PC & CF-PC & 0,28 & $-10,82$ & 0,57 & $-10,81$ & 1,20 & $-10,77$ \\
\hline
\end{tabular}



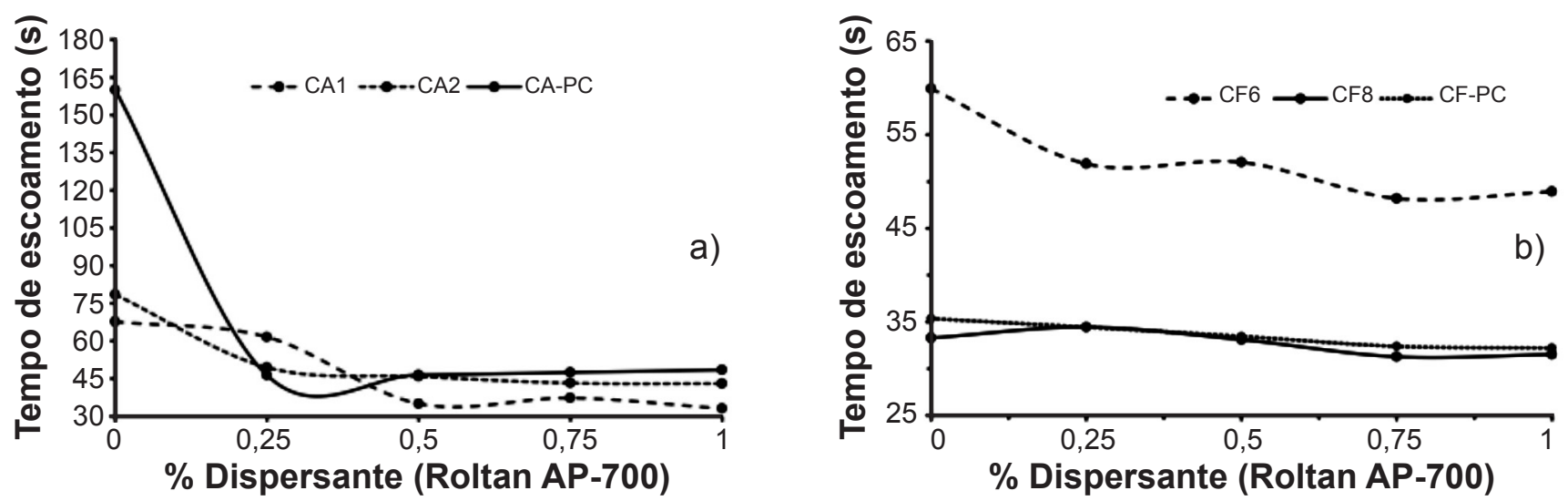

Figura 5: Tempo de escoamento das suspensões de alumina (a) e de LZSA (b) das três composições selecionadas de acordo com o teor de dispersante adicionado.

[Figure 5: Flow time of the selected alumina (a) and LZSA-based suspensions in relation to the dispersant content.]
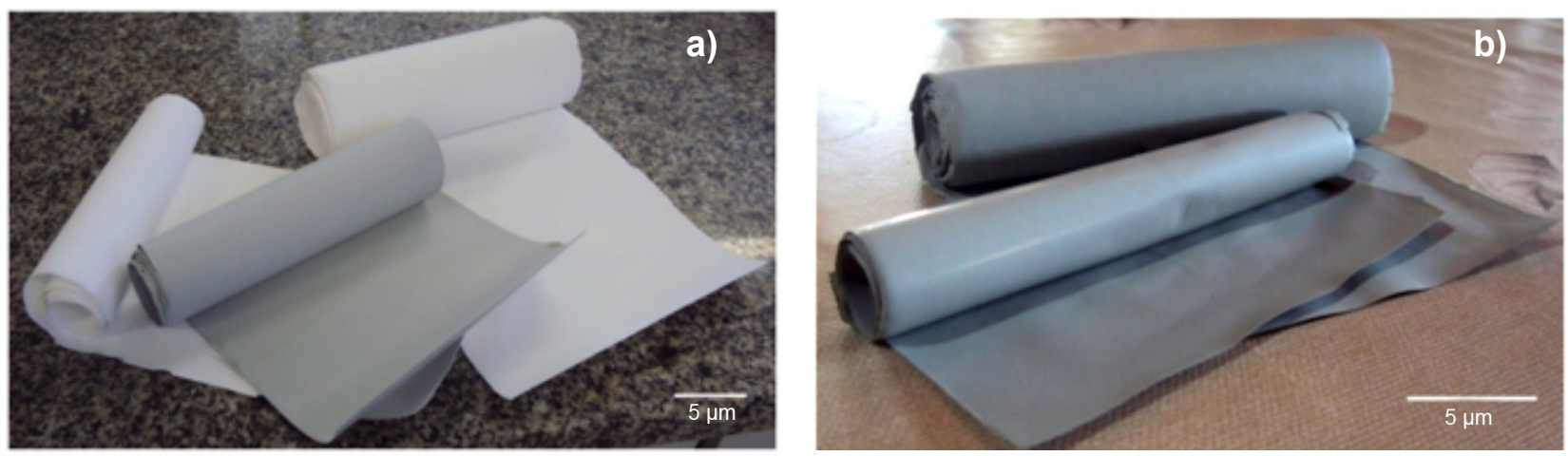

Figura 6: Imagens de: (a) folhas de alumina (para camadas de tração, externa) das três composições escolhidas (da esquerda para a direita: CA1, CA-PC e CA2); e (b) folhas da composição de LZSA CF8 (para camadas de compressão, interna).

[Figure 6: Images of: (a) tapes of the selected alumina-based compositions (for outer, tensile layers; from left to the right: CA1, CA-PC and CA2); and (b) tapes of the selected LZSA-based composition CF8 (for inner, compressive layers).]

\section{Obtenção das folhas cerâmicas}

Suspensões cerâmicas: os gráficos de tempo de escoamento versus quantidade de dispersante para as composições analisadas são mostrados na Fig. 5. Percebese na Fig. 5a uma estabilização no tempo de escoamento a partir de $0,5 \%$ de dispersante Roltan AP-700 na suspensão. Esta quantidade foi então adotada para a produção das suspensões para colagem de folhas das composições à base de alumina. Na faixa estudada para as suspensões à base de LZSA (Fig. 5b), notou-se tendência de leve diminuição do tempo de escoamento com o aumento do teor de dispersante. Paralelamente a este experimento, foi avaliada a influência do aditivo na formação de bolhas e qualidade final das folhas. Com estas avaliações, a quantidade de $1 \%$ do dispersante Roltan AP-700 foi a mais adequada e, portanto, utilizada nas suspensões para a produção das folhas cerâmicas.

Folhas cerâmicas: as folhas obtidas pela técnica de colagem de folhas, a partir das suspensões descritas, apresentaram uniformidade na espessura no sentido longitudinal, aparência homogênea, flexibilidade e resistência suficiente para o manuseio, como enrolar e cortar com tesoura ou estilete. Na Fig. 6 pode-se observar o aspecto destas folhas. A coloração cinza que pode ser observada em algumas folhas deveu-se à presença de whiskers de $\mathrm{SiC}$ nestas composições.

\section{CONCLUSÕES}

Este trabalho explorou as possibilidades de gerar compósitos cerâmicos multicamadas com interfaces fortes, partindo-se de camadas à base de alumina e vitrocerâmico do sistema $\mathrm{LZSA}\left(\mathrm{Li}_{2} \mathrm{O}-\mathrm{ZrO}_{2}-\mathrm{SiO}_{2}-\mathrm{Al}_{2} \mathrm{O}_{3}\right)$, respectivamente, para estados de tração e compressão, cujas composições foram definidas via planejamento fatorial. Os resultados indicaram que há possibilidade de estabelecimento de tensões residuais com a combinação de composições de LZSA e composições de alumina, que combinadas entre si podem formar estruturas alumina/LZSA com possibilidade de geração de tensões residuais compressivas e, possivelmente, aumento da tenacidade à fratura em relação aos respectivos monólitos. Com as três geometrias propostas, as quais, pelos cálculos executados, apresentaram tensões residuais teóricas próximas entre si para cada sistema estudado, um total de 24 possibilidades de compósito multicamada são propostos. 
Dentre as composições selecionadas, a combinação entre as composições CA1 (100\% $\mathrm{Al}_{2} \mathrm{O}_{3}$ ) e CF-PC (75\% LZSA, 5\% $\mathrm{Al}_{2} \mathrm{O}_{3}, 10 \% \mathrm{SiC}_{\mathrm{w}}$ e $\left.10 \% \mathrm{TiO}_{2}\right)$, com a geometria A composta de três camadas, foi a que demonstrou o maior potencial de geração de tensão residual de compressão na camada à base de LZSA, chegando, teoricamente, a -32,35 MPa, com tensão residual de tração associada de apenas $0,83 \mathrm{MPa}$ nas camadas de $\mathrm{Al}_{2} \mathrm{O}_{3}$. Para os cálculos, foi assumida a temperatura do último pico de cristalização do vitrocerâmico $\left(815^{\circ} \mathrm{C}\right)$ como referência, abaixo da qual as tensões se estabeleceriam entre camadas. Folhas cerâmicas (tapes) foram obtidas com sucesso a partir das suspensões de LZSA e alumina, através da técnica de tape casting com aplicação manual. As folhas obtidas são aptas ao processamento necessário para a formação dos compósitos indicados.

\section{AGRADECIMENTOS}

Os autores agradecem a CAPES (Programa CAPES/PróEstratégia 2234/2012, Processo n. 23038.008667/2012-71) pelo apoio financeiro ao desenvolvimento deste trabalho.

\section{REFERÊNCIAS}

[1] W.J. Clegg, K. Kendall, N. McN. Alford, T.W. Button, J.D. Birchall, Nature 347 (1990) 455.

[2] F.D. Minatto, P. Milak, A. De Noni Jr., D. Hotza, O.R.K. Montedo, Adv. Appl. Ceram. 114 (2015) 127.

[3] R. Bermejo, C. Baudín, R. Moreno, L. Llanes, A.J. Sánchez-Herencia, Compos. Sci. Techn. 67 (2007) 1930.

[4] N. Orlovskaya, M. Lugovy, V. Subbotin, O. Radchenko, J. Adams, M. Chheda, J. Shih, J. Sankar, S. Yarmolenko, J. Mat. Sci. 40 (2005) 5483.

[5] X. Zhang, P. Zhou, P. Hu, W. Han, J. Eur. Ceram. Soc. 31 (2011) 2415.

[6] Z. Qingfeng, D. Limin, W. Chen, C.A. Wang, H. Yong, Ceram. Int. 33 (2007) 385.

[7] G.M. Reitz, O.R.K. Montedo, O.E. Alarcon, D. Hotza,
A.P.N. Oliveira, Adv. Sci. Tech. 45 (2006) 442.

[8] O.R.K. Montedo, F.M. Bertan, R. Piccoli, D. Hotza, A.N. Klein, A.P.N. Oliveira, Am. Ceram. Soc. Bull. 87 (2008) 34. [9] O.R.K. Montedo, F.J. Floriano, J. Oliveira Filho, E. Angioletto, A.M. Bernardin, Mat. Res. 12 (2009) 197.

[10] O.R.K. Montedo, F.J. Floriano, J. Oliveira Filho, C.M. Gomes, D. Hotza, A.P.N. Oliveira, Ceram. Int. 37 (2011) 1865.

[11] O.R.K. Montedo, D. Hotza, A.P.N. Oliveira, R. Meszaros, N. Travitzky, P. Greil, Adv. Mat. Sci. Eng. (2012) 1 .

[12] O.R.K. Montedo, P. Ronsani, A.P.N. Oliveira, Química Nova 35 (2012) 689.

[13] F.M. Bertan, A.P. Novaes de Oliveira, O.R.K. Montedo, D. Hotza, C.R. Rambo, Cerâmica 59 (2013) 351.

[14] R.B Nuernberg, C.A. Faller, O.R.K. Montedo, J. Therm. Anal. Calor. 1 (2016) 1.

[15] O.R.K. Montedo, P.C. Milak, F.D. Minatto, R.B. Nuernberg, C.A. Faller, A.P.N. Oliveira, A. De Noni Jr., J. Therm. Anal. Calor. 124 (2015) 241.

[16] R. Bermejo, Y. Torres, M. Anglada, Luis Llanes, J. Am. Ceram. Soc. 91 (2008) 1618.

[17] H. Hadraba, D. Drdlik, Z. Chlup, K. Maca, I. Dlouhy, J. Cihlar, J. Eur. Ceram. Soc. 33 (2013) 2305.

[18] A. Tarlazzi, E. Roncari, P. Pinasco, S. Guicciardi, C. Melandri, G. de Portu, Wear 244 (2000) 29.

[19] M.T. Souza, F.R. Cesconeto, S. Arcaro, A.B. Teixeira, F. Raupp-Pereira, O.R.K. Montedo, A.P.N. Oliveira, J. Therm. Anal. Calor. (2016) 1.

[20] F.R. Cesconeto, S. Arcaro, F. Raupp-Pereira, J.B. Rodrigues Neto, D. Hotza, A.P.N. Oliveira, Ceram. Int. 40 (2014) 9535.

[21] R.S. Roy, H. Guchhait, A. Chanda, D. Basu, M.K. Mitra, J. Eur. Ceram. Soc. 27 (2007) 4737.

[22] Y.-F. Hsu, S.-F. Wang, Y.-R. Wang, S.-C. Chen, Ceram. Int. 34 (2008) 1183.

(Rec. 08/05/2016, Rev. 10/06/2016, 27/09/2016, Ac. 27/09/2016) 